\title{
Sample Surface Preparation For Traditional EBSD Collection and 3D EBSD Collection
}

\author{
R.E.A. Williams*, A. Genc**, D. Huber*, H.L. Fraser* \\ ** Center for the Accelerated Maturation of Materials, The Ohio State University, 2041 \\ College Rd., 477 Watts Hall, Columbus, OH 43210 \\ **Intel Inc., Hillsboro, OR 97124 \\ $\alpha / \beta$ titanium alloys posses a rich and varied two phase microstructure that consists \\ of a hcp $\alpha$ phase and a bcc $\beta$ phase. The $\alpha / \beta$ titanium microstructure is extremely \\ complex and interdependent on one another with the size scale of important \\ features ranging from millimeter to sub-micron depending on the thermo- \\ mechanical history and composition of the alloy. Titanium alloys have been applied \\ in a number of engineering applications, largely because of a combination of \\ attractive properties and relatively low density. The combination of properties that \\ may be achieved in titanium alloys are most significantly influenced by the complex \\ microstructures that can be developed in the alloys.
}

Upon typical thermo-mechanical processing the allotriomorphic $\alpha$ phase forms first heterogeneously at prior $\beta$ grain boundaries when cooling through the $\beta$ transus from the solutionizing temperature. It is well known that the laths of hcp $\alpha$ phase generally exhibit a Burgers orientation relationship with the matrix bcc $\beta$ phase. The same orientation relationship is also observed between grain boundary $\alpha$ precipitates and the matrix, however it is not possible for the allotriomorphic $\alpha$ to maintains Burgers with both $\beta$ grains across the boundary. [1,2] Understanding of this crystallographic relationship and others are critical to revealing the evolution of $\alpha / \beta$ titanium alloys. EBSD is the optimal characterization technique to probe rapidly the large scale evolution of this two phase microstructure.

In order to obtain the highest quality EBSD results it is essential to have as damage free a surface as possible. While titanium alloys tend to image rather well in backscattered electron mode, regardless of polishing technique; EBSD collection of titanium alloys can be extremely sensitive to specimen preparation. The bcc $\beta$ phase can often be impossible to index if not prepared with the utmost care. This talk will address standard mechanical polishing steps for a variety of titanium alloys that help produce an outstanding surface for EBSD collection as well as backscattered electron imaging.

With the inception of the DualBeam ${ }^{\mathrm{TM}}$ FIB and 3D EBSD, this technique quickly has proven invaluable to not only probe crystallographic data, but also the three dimensional morphology of the microstructure and interconnectivities. Currently, the technique of 3D EBSD has been successfully applied to limited metallic alloys, but not to titanium alloys due the damage created by $30 \mathrm{kV} \mathrm{Ga}^{+}$ion milling. 
Therefore in order to access the wealth of information available from 3D EBSD collection, it is critical to understand the damage layer imparted by milling with 30 $\mathrm{kV} \mathrm{Ga}+$ ions. It should be noted that his phenomena convolutes both $\alpha$ and $\beta$ EBSD patterns as well as any type of TEM analysis.

In order to analyze this damage layer a FEI Helios NanoLab ${ }^{\mathrm{TM}} 600$ has been used to contain the damage layer in cross section for analysis with a FEI Titan ${ }^{\mathrm{TM}}$ 80-300 aberration corrected microscope. HAADF STEM imaging clearly reveals the thickness of the damage layer. A technique of low $\mathrm{kV} \mathrm{Ga}^{+}$and $\mathrm{Ar}^{+}$ion milling has been implemented in order to reduce this damage layer and the marked improvement in EBSD pattern collection and TEM analysis will be presented. (Fig. 1.)

[1] T. Furuhara, et al., Metallurical and Materials Transaction, 27A (1996) 16351646

[2] T. Furuhara, et al., Materials Science and Engineering A, 312 (2001) 145-154

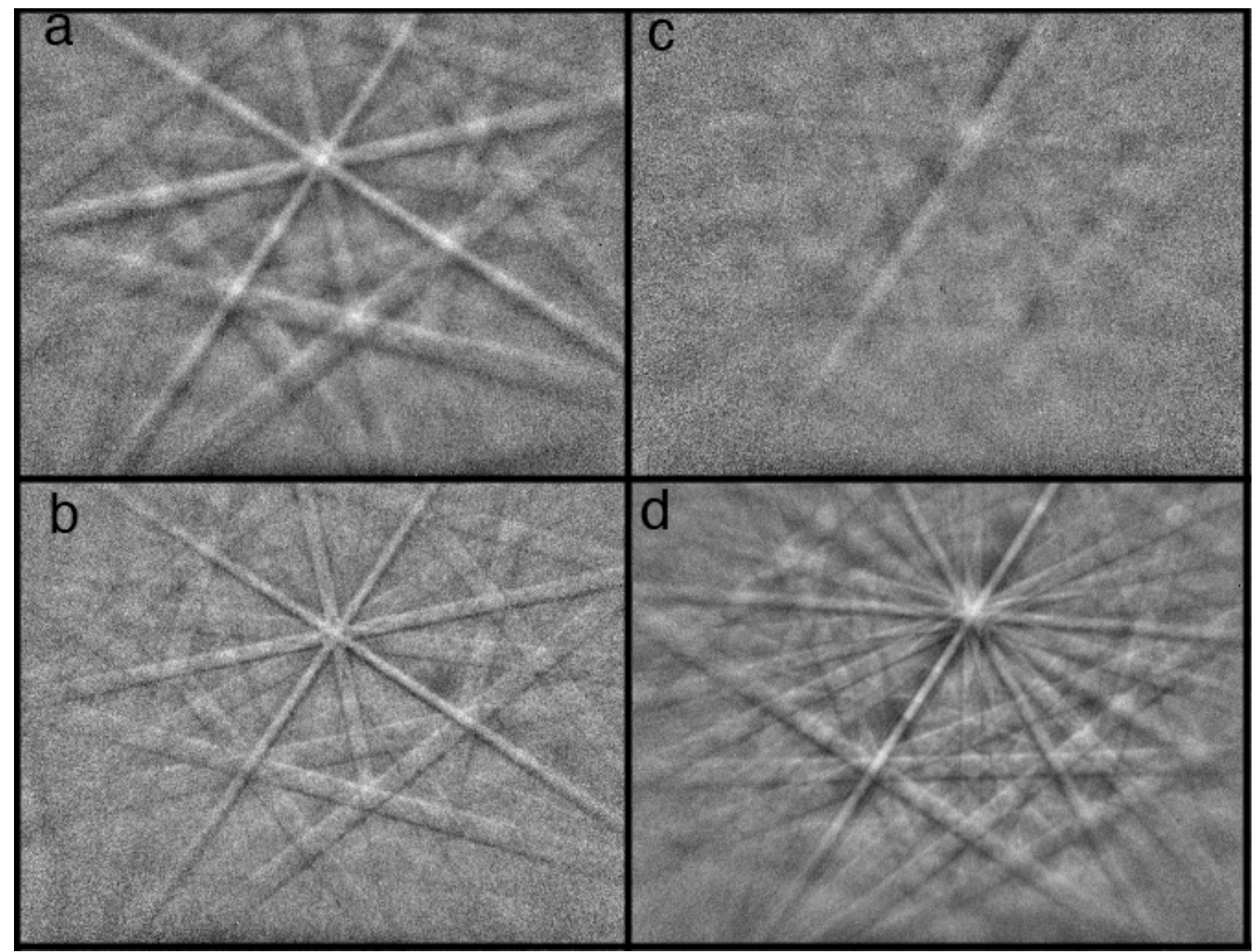

FIG. 1. EBSD patterns collected after milling with (a) $30 \mathrm{kV} \mathrm{Ga}{ }^{+}$ion of $\beta$ phase, (b) 5 $\mathrm{kV} \mathrm{Ga}+$ ion of $\beta$ phase, (c) $30 \mathrm{kV} \mathrm{Ga}+$ ion of $\alpha$ phase, (d) $5 \mathrm{kV} \mathrm{Ga}+$ ion of $\alpha$ phase. 